[15] R. B. Troxell, S. R. Kohn, J. E. Gray, R. W. Welch, E. D. Harloe, and R. K. Goyal, "A computer-assisted technique for 24-hour esophageal monitoring," Dig. Dis. Sci., vol. 27, pp. 1057-1062, 1982.

[16] J. E. Richter, W. C. Wu, D. N. Johns, J. N. Blackwell, J. L. Nelson, J. A. Castell, and D. O. Castell, "Esophageal manometry in 95 healthy adult volunteers: Variability of pressures with age and frequency of 'abnormal' contractions," Dig. Dis. Sci. . vol. 32, pp. 583$592,1987$.

\section{An Improved Buffer for Bioelectric Signals}

RAMON PALLAS-ARENY, JOSEP COLOMINAS, AND JAVIER ROSELL

\begin{abstract}
We propose an ac-coupled amplifier that offers a high input impedance, thus making it suitable for bioelectric signal amplification. We also present the necessary formulas for calculating its input impedance and transfer function in order to facilitate its adaptation to different applications.
\end{abstract}

\section{INTRODUCTION}

The amplification of bioelectric signals requires them to be ac coupled before high gain amplification, either to avoid saturations due to contact potentials arising at electrode-electrolyte interfaces [1] or for further band-limiting purposes. At the same time, in order to reduce interferences due to the usually high electrode-skin impedance and its variability [2], the amplifier needs to have a very high input impedance. This calls for an input stage acting as buffer.

In most designs, each one of these functions-ac coupling and buffering-is implemented at different stages of the circuit. In this work, we prove, both analytically and experimentally, that it is possible to merge both functions in a single stage. We also present the formulas enabling us to apply the proposed circuit to different signals in order to tailor it to particular needs.

\section{AC Coupling and InPut Impedance in Bioelectric} SigNAL AMPLIFIERS

\section{A. AC Coupling}

In order to measure bioelectric signals, it is necessary to use electrodes. At any electrode-electrolyte interface, a contact potential arises, whose magnitude is very high and subject to variations due to many parameters [1]. Thus, in spite of two electrodes being involved in any biopotential measurement, the prospect of a tight balance between them is so remote that, when designing the amplifier, the possible presence of a dc potential higher in magnitude than the expected signal must be taken into consideration. The measurement is certainly a differential one, but contact potentials would seldom cancel each other out.

The usual solution is to decouple dc voltages to prevent offset potentials from saturating the amplifier whenever its voltage gain has a value of $40 \mathrm{~dB}$ or even lower. This need of an ac coupling is a serious drawback for various reasons. In low-noise design, for example, it is important to have as much gain as possible concentrated in the first stage of the amplifier. Bioelectric signals normally require voltage gains in excess of $60 \mathrm{~dB}$.

Therefore, if ac coupling cannot be implemented before the first amplifier, as happens with ordinary circuits, the designer is prevented from taking advantage of the improved performances of

Manuscript received March 8, 1988; revised September 26, 1988.

The authors are with the Division de Instrumentacion y Bioingenieria, Departamento de Ingeniería Electrónica, U.P.C., ETS Ing Telecom, P.O. Box 30002,08080 Barcelona, Spain.

IEEE Log Namber 8825623.

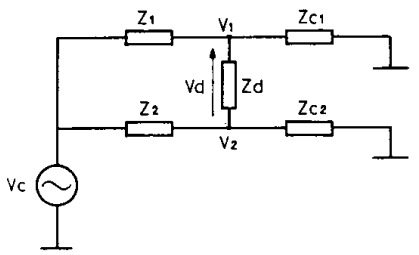

Fig. 1. Circuit model for the input of a biopotential amplifier showing how any imbalance in the front end converts a common mode signal into a differential one. The imbalance can be on the electrode side or on the amplifier side.

modern operational amplifiers which offer very low noise levels because he or she is compelled to postpone high-voltage gains until an ac signal is obtained [3]. Furthermore, for a given tolerance in components, the CMRR attained increases if voltage gain is large in differential stages, although it is unaffected by voltage gain in nondifferential ones.

Also, when dealing with several electrodes and only one or a few differential amplifiers, it is necessary to place a buffer for each electrode. If they are dc coupled, it may happen that a saturation occurs in the amplifier when switching from one lead to another because of electrode offset potentials. Therefore, some provision must be made in order to rapidly restore voltage levels. An accoupled buffer might eliminate this problem.

\section{B. Input Impedances}

Another requirement when measuring bioelectric signals is the need for differential amplifiers to have very high input impedances, both differential and common mode. A high differential mode input impedance minimizes loading effects. A high common mode input impedance reduces the differential mode voltage that results from common mode input signals [2]. This common mode-to-differential mode conversion results from any imbalance between both input terminals, as indicated in Fig. 1, and shows up as an increased interference at power line frequency.

In Fig. $1, Z_{1}$ and $Z_{2}$ are any impedances in front of the amplifier. Whenever either $Z_{1} \neq Z_{2}$ or $Z_{c 1} \neq Z_{c 2}$, the former being the usual situation, then $v_{1} \neq v_{2}$, and an interfering signal will result. In most designs, $Z_{1}$ and $Z_{2}$ are mainly attributable to electrode-skin impedances. The resulting interference can be effectively reduced by making $Z_{c 1}$ and $Z_{c 2}$ very high. Buffering each electrode with a unity gain voltage follower or a buffer with gain is the usual solution.

The placement of a capacitor in series with each electrode would certainly block any dc voltage, and therefore might be considered for decoupling. However, there is a simultaneous need for a path for $\mathrm{dc}$ bias currents entering the amplifier, and the blocking capacitor would prevent any dc current from coming through the electrodes. Placing a resistor from each input of the amplifier to ground would solve this problem, but it would be necessary to use rather unusual components in order to obtain the high input impedance required for a bearable interference and the optimum low-frequency response.

For these reasons, typical biopotential amplifiers have a dc-coupled moderate-gain input stage, thus biasing through the electrodes and reducing the risk of saturation at this stage. Afterwards-preferably once dealing with a nondifferential signal-an ac-coupled stage offering an additional gain is put in place. A typical circuit is the one proposed by Neuman [4].

\section{ANalysis of an AC-Coupled Buffer}

In accordance with the above reasons, the placement of a series capacitor in front of an amplifier will be a satisfactory solution, provided that a suitable way of biasing the circuit is offered. This 


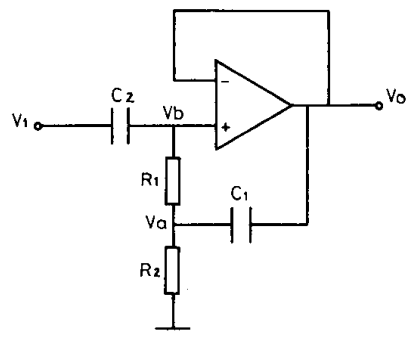

Fig. 2. Proposed ac-coupled buffer. A series capacitor blocks dc currents.

means that unusual resistors need not be required and that input impedances must be kept high in any case.

Thakor and Webster [5] proposed a double-ended two-electrode design based upon a bootstrapped operational amplifier as an input stage. This stage provided a high common mode input impedance and a path for the dc bias current of the operational amplifier without using high-value resistors. The effective input impedance increased with frequency and was very high at power line frequency. A refined design was later presented by Winter and Webster [2].

On the face of it, it would seem that by merely placing a suitable capacitor in series with the input of such a circuit, an ac-coupled buffer will be obtained. The resulting circuit is the one in Fig. 2. The validity of this assumption, however, must be checked by examining both the characteristics we are interested in, that is, the transfer function and input impedance.

\section{A. Transfer Function}

The relationship between $v_{0}$ and $v_{1}$ can be found by combining the following equations, deduced from a simple inspection of the circuit in Fig. 2:

$$
\begin{aligned}
C_{2} s\left(v_{1}-v_{b}\right) & =\left(v_{b}-v_{a}\right) / R_{1} \\
\left(v_{b}-v_{a}\right) / R_{1}+\left(v_{0}-v_{a}\right) C_{1} s & =v_{a} / R_{2} \\
\left(v_{b}-v_{0}\right) A_{0} \omega_{a} /\left(s+\omega_{a}\right) & =v_{0}
\end{aligned}
$$

where $A_{0}$ is the open-loop gain of the operational amplifier and $\omega_{a}$ is its corner frequency. Given that $A_{0} \gg 1$, the desired relationship proves to be

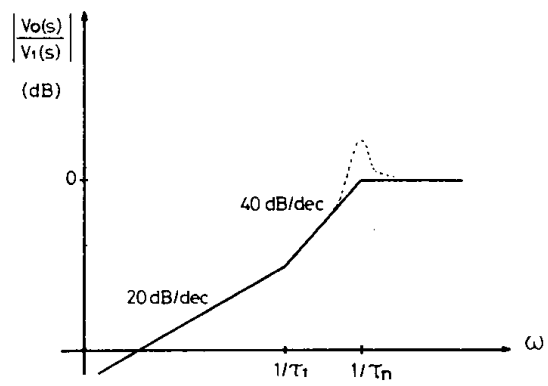

(a)

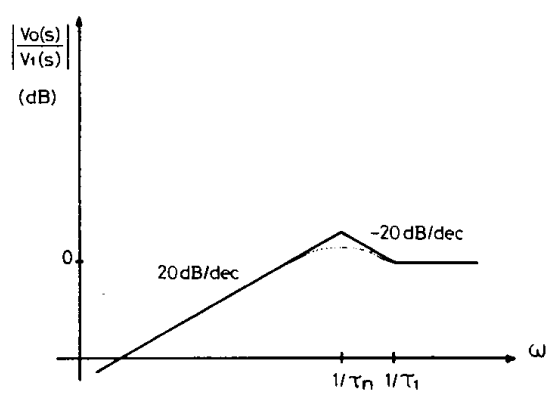

(b)

Fig. 3. Frequency characteristic of circuit in Fig. 2-see (5). Depending on the relative value of the components, two cases, (a) $\tau_{1}>\tau_{n}$, (b) $\tau_{1}$ $<\tau_{n}$ will result. A flat frequency response is not granted in all cases. The frequency where there is the maximum amplitude is given by (14) and the maximum amplitude is given by (15). Corner frequency ( -3 $\mathrm{dB})$ is given by $(16)$.

The damping factor $\epsilon$ and the natural frequency $\omega_{n}$ are given, respectively, by

$$
\begin{aligned}
\epsilon & =\left(\tau_{2} / \tau_{1}\right)^{1 / 2} / 2 \\
w_{n} & =\left(1 / \tau_{1} \tau_{2}\right)^{1 / 2}=1 / \tau_{n} .
\end{aligned}
$$

$$
\frac{V_{0}(s)}{V_{1}(s)}=\frac{s \tau_{2}\left(1+\tau_{1} s\right)}{1+s\left[\tau_{2}+\left(\tau_{m}+\tau_{a}\right) / A_{0}\right]+s^{2}\left[\tau_{n}^{2}+\tau_{a}\left(\tau_{m}+\tau_{2}\right) / A_{0}\right]+s^{3} \tau_{n}^{2} \tau_{a} / A_{0}}
$$

where

$$
\begin{aligned}
\tau_{2} & =\left(R_{1}+R_{2}\right) C_{2} \\
\tau_{1} & =\left(R_{1} / / R_{2}\right) C_{1} \\
\tau_{m} & =R_{2} C_{1} \\
\tau_{a} & =1 / \omega_{a} \\
\tau_{n}^{2} & =R_{1} R_{2} C_{2} C_{1}=\tau_{2} \tau_{1} .
\end{aligned}
$$

For high-frequency signals, expression (4) indicates that there is an increasing attenuation. The circuit will behave as a unity gain voltage follower only at low frequencies, excluding dc.

For low-frequency signals, the circuit time constants can be designed in such a way that all the terms including $A_{0}$ in the denominator become negligible. If this is the case, that is, for frequencies below $A_{0} /\left(\tau_{m}+\tau_{a}\right)$, below $\left[A_{0} /\left(\tau_{m}+\tau_{2}\right) \tau_{a}\right]^{1 / 2}$, and below $\left[A_{0} / \tau_{n}^{2} \tau_{a}\right]^{1 / 3}$, then expression (4) can be simplified to

$$
\frac{V_{0}(s)}{V_{1}(s)}=\frac{s\left(s+1 / \tau_{1}\right)}{s^{2}+s / \tau_{1}+1 / \tau_{1} \tau_{2}} .
$$

Thus, while the frequency response will always be high pass at low frequencies, its shape will depend on the relative magnitude of $\tau_{1}$ and $\tau_{n}$, as indicated in Fig. 3 .

The decision about whether it is better to have $\tau_{1}$ greater, lower, or equal to $\tau_{2}$ cannot be taken, however, before examining its effect on the input impedance.

\section{B. Input Impedance}

The input impedance for the circuit in Fig. 2 is given by

$$
Z=\frac{v_{1}}{\left(v_{1}-v_{b}\right) C_{2} s} .
$$

After some manipulations of expressions (1)-(3), and given that $A_{0}$ $\gg 1$, the following is obtained:

$$
Z=\frac{1}{C_{2} s}\left(1+\frac{s \tau_{2}+s^{2}\left(\tau_{n}^{2}+\tau_{u} \tau_{2} / A_{0}\right)+s^{3} \tau_{n}^{2} \tau_{a} / A_{0}}{1+s\left(\tau_{m}+\tau_{u}\right) / A_{0}+s^{2} \tau_{a} \tau_{m} / A_{0}}\right) .
$$




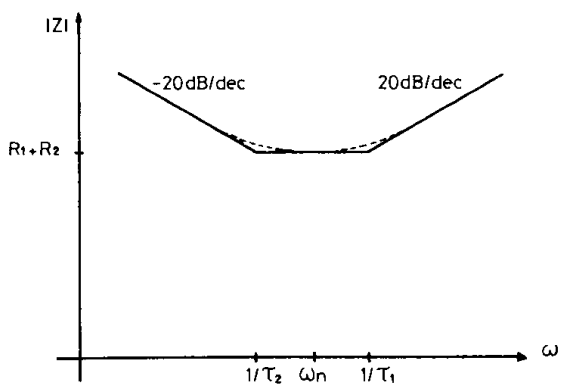

(a)

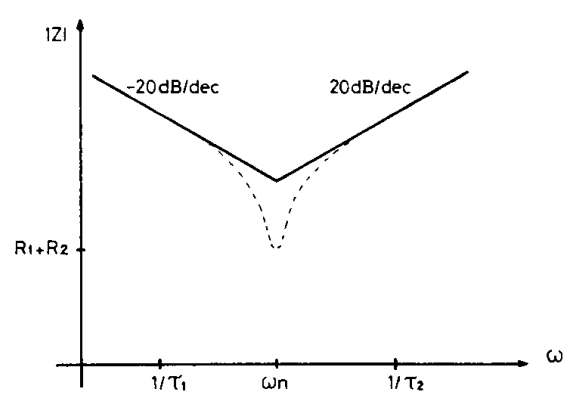

(b)

Fig. 4. Input impedance of circuit in Fig. 2-see (11). Depending on the relative value of the components, two cases, (a) $\tau_{1}<\tau_{2}$, (b) $\tau_{1}>\tau_{2}$ will result. A high input impedance is not granted in all cases.

Here, too, if only the low-frequency part of the spectrum is considered, the following simplified formula is obtained:

$$
Z=\frac{1}{C_{2} s}\left(1+s \tau_{2}+s^{2} \tau_{1} \tau_{2}\right)
$$

and by replacing time constants by their expressions, the following results:

$$
Z=\frac{1}{C_{2} s}+R_{1}+R_{2}+s R_{1} R_{2} C_{1}
$$

This is the same result as that obtained by Winter and Webster [2] with an additional term in our case contributed by series capacitor $C_{2}$. Fig. 4 shows this input impedance as a function of frequency for the cases $\tau_{2}>\tau_{1}$ and $\tau_{2}<\tau_{1}$.

\section{Design of an AC-Coupled Buffer}

An appropriate ac-coupled buffer must simultaneously have a flat frequency response and a very high input impedance. In order to ensure a high input impedance at power line frequency and its harmonics, without using high-value resistors, it is necessary for these frequencies to fall within the zone where $Z$ looks like an inductor, that is,

$$
R_{1}+R_{2}<\omega_{i} R_{1} R_{2} C_{1}
$$

where $\omega_{i}$ is the frequency of the interfering signal considered.

The frequency response in the passband must be flat according to some criterion. The American Heart Association, for example, recommends a response for direct-writing electrocardiographs which is flat to within $\pm 0.5 \mathrm{~dB}$ over the frequency range from 0.14 to $25 \mathrm{~Hz}$. Therefore, it is necessary to ensure both that at the lower frequency of interest, the attenuation, if any, does not exceed 0.5 $\mathrm{dB}$, and that the damping factor in (5) has a value which does not lead to overshooting in the frequency response.

By equating the numerator and denominator modulus in (5), it can easily be deduced that the modulus of the transfer function takes a value of 1 at a frequency $\omega_{0}$ given by

$$
\omega_{0}=\omega_{n} / \sqrt{2} \text {. }
$$

At the same time, by observing the approximated transfer function for $\omega \gg \omega_{n}$, it is concluded that the modulus is also 1 at frequencies well above $\omega_{n}$.

Therefore, a second condition to be considered when designing such a buffer is for the minimum frequency of interest not to be lower than $\omega_{0}$. It is important to observe that this condition is independent of the damping factor.

The transfer function (5) has a maximum amplitude at a frequency $\omega_{r}$ given by

$$
\omega_{r}=\omega_{n}\left[1+\left(1+8 \epsilon^{2}\right)^{1 / 2}\right]^{1 / 2} / \sqrt{2} .
$$

The magnitude of this maximum is given by

$$
\left|\frac{V_{0}(s)}{V_{1}(s)}\right|^{2}=\frac{1+8 \epsilon^{2}+\left(4 \epsilon^{2}+1\right)\left(1+8 \epsilon^{2}\right)^{1 / 2}}{1+8 \epsilon^{2}+\left(4 \epsilon^{2}-1\right)\left(1+8 \epsilon^{2}\right)^{1 / 2}}
$$

By subtracting 1 to the square root of this expression, the magnitude of the overshooting in the frequency response is obtained. It has been represented in Fig. 5 as a function of $\epsilon$. According to the latter, if the maximum deviation allowed with respect to a flat response is of $0.5 \mathrm{~dB}$, then $\epsilon$ must be higher than 1.76 .

The value of $\epsilon$ will influence the rate of attenuation at frequencies below $\omega_{0}$. The frequency $\omega_{m}$ at which there is a $3 \mathrm{~dB}$ attenuation is easily deduced from (5) to be

$$
\omega_{m}=\omega_{n}\left\{\left[\left(2 \epsilon^{2}+1\right)^{2}+1\right]^{1 / 2}-\left(2 \epsilon^{2}+1\right)\right\}^{1 / 2} .
$$

Therefore, the lower the value of $\epsilon$, the higher the value of $\omega_{m}$, as shown in Fig. 5 (right vertical axis).

Example: Let us suppose that a buffer for the ECG signal is desired, with an input impedance equal to or higher than $100 \mathrm{M} \Omega$ at $50 \mathrm{~Hz}$, a flat frequency response $( \pm 0.5 \mathrm{~dB})$ from $0.14 \mathrm{~Hz}$, and without using resistors higher in value than $10 \mathrm{M} \Omega$.

From (12) and (13), it is deduced that

$$
R_{1} R_{2} C_{1} 100 \pi=10^{8} \text {. }
$$

From (13), it follows

$$
2 \pi 0.14=\frac{1}{\left(2 R_{1} R_{2} C_{1} C_{2}\right)^{1 / 2}} .
$$

If a maximum attenuation is desired for frequencies below $0.14 \mathrm{~Hz}$, then according to Fig. 5 and (16), $\epsilon$ must be 1.76. From (6), it follows that

$$
\left(R_{1}+R_{2}\right)^{2} C_{2}=12.4 R_{1} R_{2} C_{1} .
$$

Expressions (17) and (18) determine $C_{2}=2 \mu \mathrm{F}$. Expressions (17) and (19) determine the condition $R_{1}+R_{2}=1.4 \mathrm{M} \Omega$. If, in order to use resistors with the lowest possible value, the additional condition $R_{1}=R_{2}=R$ is imposed, then $R=720 \mathrm{k} \Omega$ and from (17), $C_{1}=650 \mathrm{nF}$.

We measured the transfer function and input impedance of a circuit such as that in Fig. 2 implemented with $R_{1}=R_{2}=680 \mathrm{k} \Omega$, tolerance 5 percent, $C_{1}=680 \mathrm{nF}$, and $C_{2}=2 \mu \mathrm{F}$, tolerance 10 percent. The operational amplifier was a TL071 and the circuit was arranged in order to minimize parasitic capacitances. The following numerical results were read from the display on a HewlettPackard Spectrum Analyzer 3582A: minimum frequency where gain was $0 \mathrm{~dB}, 0.16 \mathrm{~Hz}$; frequency of $-3 \mathrm{~dB}$ gain, $0.052 \mathrm{~Hz}$; and maximum gain, $0.4 \mathrm{~dB}$ at frequencies between 0.288 and $0.56 \mathrm{~Hz}$. By calculation, the following results were deduced: input impedance at $50 \mathrm{~Hz}, 141 \mathrm{M} \Omega$; maximum input impedance, 1,63 $\mathrm{G} \Omega$ at $124 \mathrm{~Hz}$.

The preceding results show that the input impedance at $50 \mathrm{~Hz}$ is higher than predicted, but by making allowance for the tolerances of the components, it can be accepted. Yet the decreasing of the impedance starting at $124 \mathrm{~Hz}$ cannot be explained by the model 


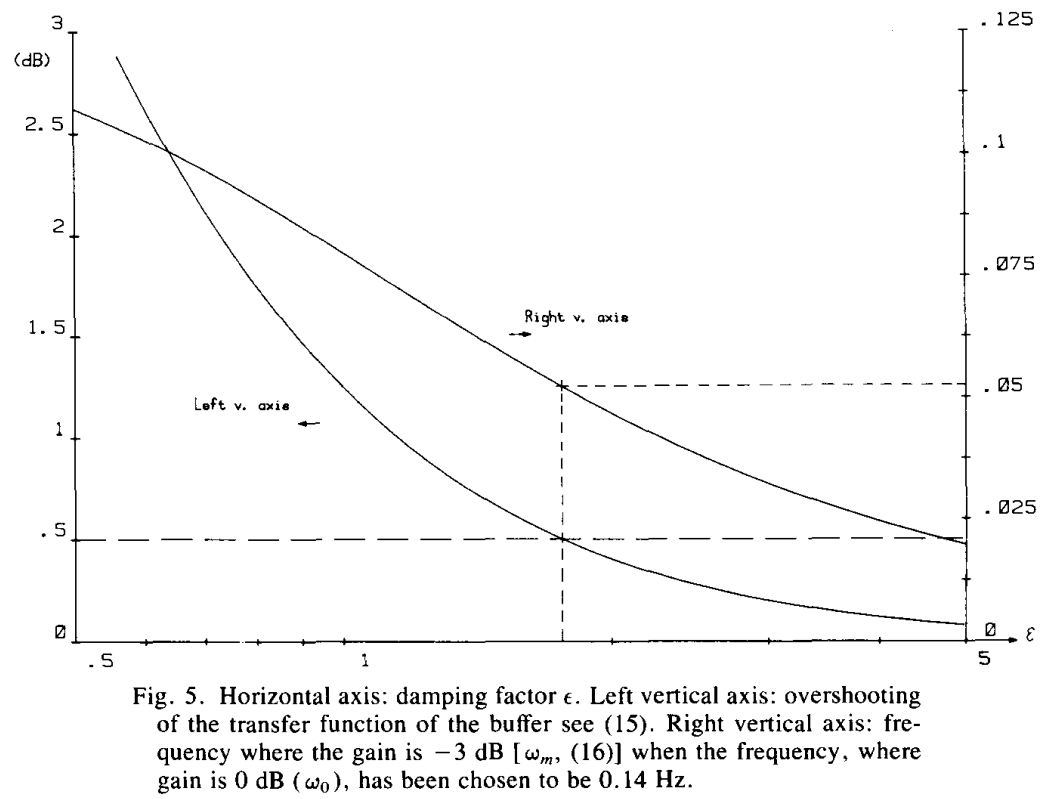

used for the circuit because gain limitations of the operational amplifier do not have any noticeable effect at these low frequencies.

The fact that the impedance at $124 \mathrm{~Hz}$ is 11.5 times that at 50 $\mathrm{Hz}$, instead of 2.5 times as predicted by an inductive-looking impedance, suggests that there is some resonance effect. Assuming this, if an input capacitance $C$ paralleling the equivalent input inductor $L$ is considered, then from the value of the impedance at 50 and $124 \mathrm{~Hz}$, it is deduced that $C \approx 3 \mathrm{pF}$ and $L \approx 0.4 \mathrm{MH}$. At 50 $\mathrm{Hz}$, this inductance has an impedance of $120 \mathrm{M} \Omega$. The frequency of resonance is near $145 \mathrm{~Hz}$.

\section{Conclusions}

By adding a single capacitor to a well-known dc-coupled buffer, it is feasible to obtain an ac-coupled buffer with very high inpu impedance without resorting to high-value resistors. The necessary formulas for adapting this circuit to any particular need when dealing with low-frequency signals are relatively simple. By using the appropriate equations, it is possible to choose the value of the input impedance, the maximum overshooting in the frequency response. and the "low corner" frequency.

The effective input impedance will, in fact, be limited by common mode input capacitances associated not only with the operational amplifier, but also with input protections, RF filters, and circuit layout. These capacitances will, however, provide an increased impedance near the frequency of resonance.

\section{REFERENCES}

[1] M. R. Neuman, "Biopotential electrodes," in Medical Instrumenta tion Application and Design, J. G. Webster, Ed. Boston, MA Houghton Mifflin, 1978, ch. 5

[2] B. B. Winter and J. G. Webster, "Reduction of interference due to common mode voltage in biopotential amplifiers," IEEE Trans. Biomed. Eng., vol. BME-30, pp. 58-62, 1983.

[3] R. van Heuningen, H. G. Goovaerts, and F. R. de Vries, "A low noise isolated amplifier system for electrophysiological measurements: Basic considerations and design," Med. Biol. Eng. Comput., vol. 22, pp. $77-85,1984$.

[4] M. R. Neuman, "Biopotential amplifiers," in Medical Instrumentation Application and Design, J. G. Webster, Ed. Boston, MA Houghton Mifflin, 1978 , ch. 6, p. 309

[5] N. V. Thakor and J. G. Webster, "Ground free ECG recording with two electrodes," IEEE Trans. Biomed. Eng., vol. BME-27, pp. 699704,1980

\section{Two-Dimensional Fourier Spectrum of QRST Integral Maps in Classification of Patients Prone to Ventricular Arrhythmia}

\author{
SHALINI S. PERIYALWAR, SHERWIN T. NUGENT, \\ AND B. MILAN HORACEK
}

Abstract-Two-dimensional Fourier spectra of $Q R S T$ integral maps, obtained by body surface potential mapping, were analyzed to identify subjects prone to ventricular arrhythmia, when they have not been identified by the extrema count method. The diagnostic performance ( 84.38 percent) of the peak value of the Fourier spectrum as a classifier for subjects prone to ventricular arrhythmia showed an improvement of 3.65 percent over the use of the extrema count method as a classifier.

\section{INTRODUCTION}

Body surface potential maps have been found to reveal more information about the electrical activity of the heart than can be found from conventional electrocardiograms [1], [2]. The extensive amount of data involved in a total body surface potential map permits its visual analysis only in a global fashion. The maps are qualitatively analyzed in terms of simple parameters such as the location of maxima and minima [3], [4]. The application of analytical representation techniques to body surface potential mapping allows visual study of the maps in an orthogonal or transform domain and the assessment of the maps on the basis of other criteria; for example, these techniques have been applied to the determination of potentials at sites between electrodes [5].

Manuscript received March 28, 1988; revised October 10, 1988. This work was supported by grants from the Natural Science and Engineering Research Council of Canada and the Nova Scotia Heart Foundation.

S. S. Periyalwar is with the Department of Electrical Engineering, Technical University of Nova Scotia, Halifax, N.S. Canada B3J 2 X4

S. T. Nugent is with the Department of Engineering, Dalhousie University, Halifax, N.S. Canada B3H 3 J5.

B. M. Horacek is with the Department of Physiology and Biophysics, Dalhousie University, Halifax, N.S. Canada B3H $4 \mathrm{H} 7$.

IEEE Log Number 8825622. 